\title{
On the Stability of Coagulation-Fragmentation Population Balances
}

\author{
R. DENNIS VIGIL ${ }^{1}$ AND ROBERT M. ZIFF \\ Department of Chemical Engineering, The University of Michigan, Ann Arbor, Michigan 48109
}

Received December 16, 1988; accepted March 2, 1989

\begin{abstract}
When coagulation and fragmentation both occur in a system, the competition between these processes may lead to a steady-state size distribution. We consider some specific moment solutions to a generalized coagulation-fragmentation population balance equation (in which multiple breakup is allowed) in order to determine when it is may be possible for such steady states to exist. Steady states occur for systems with homogeneous rate kernels of order $\beta$ (fragmentation) and $\lambda$ (coagulation) that satisfy $\beta-\lambda+1$ $>0$. Finally, we discuss the applicability of scaling to this generalized coagulation-fragmentation population balance. (c) 1989 Academic Press, Inc.
\end{abstract}

\section{INTRODUCTION}

The study of coagulation and fragmentation processes is relevant to such diverse fields as colloidal chemistry, aerosol science, and polymer science. A major objective in these studies has been to understand how the microscopic interactions between objects lead to the observable macroscopic behavior. Population balance equations (PBE's), first applied to irreversible colloidal coagulation by Smoluchowski (1), provide the mathematical framework for describing macroscopic behavior (the evolution of the particle size distribution, polydispersity) given particle reaction rates.

Analytical solutions to Smoluchowski's coagulation equation, given in its continuous formulation by

$$
\begin{aligned}
& \frac{\partial c(x, t)}{\partial t}= \\
& \frac{1}{2} \int_{0}^{x} K(y, x-y) c(y, t) c(x-y, t) d y \\
& \quad-c(x, t) \int_{0}^{\infty} K(x, y) c(y, t) d y,
\end{aligned}
$$

have been found for the three simple rate kernels $K(x, y)=a, a(x+y)$, and $a x y$, where

\footnotetext{
${ }^{1}$ To whom all correspondence should be addressed.
}

$c(x, t)$ is the concentration of $x$-sized particles at time $t$ and $a$ is a constant (1-4). The solution for the constant kernel, $K(x, y)=a$, has found applications for both Brownian coagulation and the growth of linear polymers while the sum kernel, $K(x, y)=a(x+y)$, is relevant to branched polymerization processes and to aerosol coagulation in either laminar or turbulent flow $(2,3)$. The product kernel, which has been applied to the kinetics of growth for cross-linked polymers, has been shown to lead to a phase transition known as gelation in which mass is lost to a new phase composed of an infinite-sized particle $(5,6)$.

The problem of irreversible fragmentation has received attention primarily in connection with the study of polymer degradation (7-10), grinding and crushing (11-13), and char combustion $(14,15)$. A general fragmentation PBE that describes these processes is given by

$$
\begin{aligned}
\frac{\partial c(x, t)}{\partial t}= & -a(x) c(x, t) \\
& +\int_{x}^{\infty} a(y) b(x \mid y) c(y, t) d y,
\end{aligned}
$$

where $a(x)$ is the overall rate of breakup of $x$ sized particles and $b(x \mid y)$ is a distribution function describing the probability of producing an $x$-sized particle from the breakup of a 
$y$-sized particle. For most physical problems, it has been found that the overall rate of breakup has the power-law form $a(x)=k x^{\beta}$, where $k$ is a constant (12). Equation [2] has been solved explicitly for $a(x)=k x^{\beta}$ and either $b(x \mid y)=f(y) x^{\nu},-2<\nu \leqslant 0$ (powerlaw breakup) or $b(x \mid y)=g(y) x^{\beta-2}(y-x)$ (parabolic breakup), where the functions $f(y)$ $=(\nu+2) / y^{\nu+1},(\nu>-2)$ and $g(y)=(\beta+2)(\beta$ $+1) / y^{\beta+1}$ are determined by conservation of mass $(16,17)$. For cases in which $\beta<0$, these fragmentation kernels lead to a phase transition analogous to gelation and known as "shattering," in which mass in the system is lost to a new phase of zero-sized particles (13, 17). In such cases, the number of particles in the system becomes infinite and the mass of finite-sized particles is not conserved.

Recently, the more general problem of simultaneous coagulation with fragmentation has begun to receive more attention. Family et al. (18) performed computer simulations of coagulation with binary fragmentation and found a scaling law $S(t=\infty, k) \sim k^{-Y}$ as $k \rightarrow 0$, where $S$ is the mean particle size, $k$ is a constant representing the relative strength of fragmentation to coagulation, and $Y$ is related to the homogeneity exponents of the coagulation and breakup kernels. Sorensen et al. (19) applied the scaling form for nongelling irreversible coagulation (20) to a coagulationbinary fragmentation PBE and derived a criterion for determining the existence of steadystates. However, it has subsequently been shown that their scaling conjecture is incorrect for coagulation-fragmentation (21-22), except in the case of the well-known Blatz-Tobolsky model (23). Most recently, Meakin and Ernst (22) have suggested a two-parameter scaling equation for coagulation with binary fragmentation and have performed computer simulations that verify this scaling law in three dimensions and at steady state in one and two dimensions.

In this paper we consider a general coagulation-multiple fragmentation PBE and investigate the existence of steady-state solutions to this equation for a wide range of coagulation and fragmentation kernels. We then discuss the applicability of scaling solutions to these equations.

\section{STABILITY OF COAGULATION- FRAGMENTATION PBE}

Combination of [1] and [2] yields the generalized coagulation-fragmentation equation

$$
\begin{aligned}
& \frac{\partial c(x, t)}{\partial t}= \\
& \frac{1}{2} \int_{0}^{x} K(y, x-y) c(y, t) c(x-y, t) d y \\
& -c(x, t) \int_{0}^{\infty} K(x, y) c(y, t) d y-a(x) c(x, t) \\
& \quad+\int_{x}^{\infty} a(y) b(x \mid y) c(y, t) d y
\end{aligned}
$$

We call [3] a generalized equation because it includes multiple fragmentation, in contrast to most other works where only binary fragmentation is allowed. Explicit, time-dependent solutions to [3] are previously unknown, except for a few specific cases applicable to polymer growth $(23,24)$. In general a system described by [3] cannot reach a dynamic equilibrium in which detailed balance is satisfied, since multiple product fragments from a single breakup event are allowed. However, steady states may still exist if $\partial c(x, t) / \partial t=0$ has a solution. In this paper we refer to systems described by [3] which have steady-state solutions as stable systems and to those that have no steady-state solutions as unstable systems.

The moments of the size distribution, $M_{n}$ $\equiv \int_{0}^{\infty} x^{n} c(x, t) d x$, are obtained from [3] for the power-law fragmentation kernel by multiplying by $x^{n}$, inserting the relations $a(x)$ $=k x^{\beta}, b(x \mid y)=(\nu+2) x^{\nu} / y^{\nu+1}$, and integrating

$$
\begin{aligned}
\frac{d M_{n}}{d t}= & \frac{1}{2} \int_{0}^{\infty} \int_{0}^{\infty} K(x, y) c(x, t) c(y, t) \\
& \times\left[(x+y)^{n}-x^{n}-y^{n}\right] d x d y \\
& +k\left(\frac{1-n}{n+\nu+1}\right) M_{n+\beta}
\end{aligned}
$$


where $k$ is a nonnegative constant which gives the relative strength of fragmentation to coagulation. We give explicit solutions to [4] for $n=0$ and/or 2 and for $\beta=1,0,-1$ in combination with each of the three basic coagulation kernels (nine cases altogether) as outlined below.

(1) $\beta=-1, K(x, y)=1$. The second term of Eq. [4] couples to the $(n-1)$-th moment, so a closed equation for $M_{0}$ cannot be found. However, the second moment, $M_{2}$ couples to the first moment, $M_{1}$, which represents the total mass in the system and is conserved $\left(M_{1}\right.$ $=$ constant $\equiv 1$ ) before either shattering or gelation occurs. We find from [4] that

$$
M_{2}(t)=M_{2}(0)+\left(1-\frac{k}{\nu+3}\right) t .
$$

Equation [5] implies that the long-time behavior of $M_{2}$ depends upon the parameters $\nu$ and $k$. When $k<\nu+3, M_{2} \rightarrow \infty$ as $t \rightarrow \infty$, suggesting that coagulation will overpower fragmentation and the average particle size will grow linearly. However, if $k>\nu+3, M_{2}$ becomes negative at $t_{\mathrm{s}}=M_{2}(0) /\left(k(\nu+3)^{-1}\right.$ - 1). It is impossible for $M_{2}$ to become negative unless $M_{1}$ becomes time-dependent (indicating the formation of a new phase). Thus, we conclude that a shattering transition occurs if $k>\nu+3$.

(2) $\beta=-1, K(x, y)=x+y$. Equation [4] yields

$$
\begin{aligned}
M_{2}(t)= & \frac{k}{2(\nu+3)} \\
& \quad+\left[M_{2}(0)-\frac{k}{2(\nu+3)}\right] e^{2 t} .
\end{aligned}
$$

In contrast to case 1 above, the long-time behavior of this system does depend upon the initial conditions. When $M_{2}(0)>k / 2(\nu+3)$, $M_{2}$ grow exponentially and the breakup process is not strong enough to halt coagulation. Otherwise, $M_{2}$ becomes negative at $t_{\mathrm{s}}=(-1 /$ 2) $\ln \left[1-2(\nu+3) M_{2}(0) / k\right]$ again implying shattering.
(3) $\beta=-1, K(x, y)=x y$. For this set of kernels [4] gives

$$
M_{2}(t)=\alpha \frac{1+b e^{2(k /(\nu+3))^{1 / 2} t}}{1-b e^{2(k /(\nu+3))^{1 / 2} t}},
$$

where $\alpha \equiv[(\nu+3) / k]^{-1 / 2}$ and $b \equiv\left(M_{2}(0)\right.$ $-\alpha) /\left(M_{2}(0)+\alpha\right)$. If $M_{2}(0)>\alpha$, then $M_{2}$ diverges at $t_{\mathrm{g}}=(1 / 2) \alpha^{-1 / 2} \ln \left[\left(M_{2}(0)+\alpha\right) /\right.$ $\left.\left(M_{2}(0)-\alpha\right)\right]$, indicating that gelation occurs. However, if $M_{2}(0)<\alpha$, then $M_{2}$ becomes negative at $t_{\mathrm{s}}=(1 / 2) \alpha^{-1 / 2} \ln \left[\left(\alpha+M_{2}(0)\right) /\right.$ $\left.\left(\alpha-M_{2}(0)\right)\right]$ implying shattering.

(4) $\beta=0, K(x, y)=1$. In this case particles fragment at a rate independent of their sizes. This is the borderline case before shattering can occur. Note that when $\beta=0$ the moment equation [4] decouples from higher order terms and, in principle, all of the moments may be found. Here, we give solutions for the zeroth and second moments:

$$
\begin{aligned}
M_{2}(t) & =\frac{(\nu+3)}{k} \\
& +\left[M_{2}(0)-\frac{(\nu+3)}{k}\right] e^{-k t /(\nu+3)}
\end{aligned}
$$

$M_{0}(t)$

$$
=\frac{2 k M_{0}(0)}{(\nu+1)\left[M_{0}(0)-\left[M_{0}(0)\right.\right.}-.
$$

Since conservation of mass requires that $\nu$ $>-2$, this system is always stable since it approaches the steady-state $M_{2} \rightarrow(\nu+3) / k$ as $t \rightarrow \infty$. Similarly, $M_{0}$, which is only defined for $-1<\nu \leqslant 0$, approaches a steady state given by $M_{0} \rightarrow 2 k /(\nu+1)$ as $t \rightarrow \infty$.

(5) $\beta=0, K(x, y)=x+y$. For these kernels we find

$$
\begin{aligned}
& M_{2}(t)=M_{2}(0) e^{(2-k /(\nu+3)) t} \\
& M_{0}(t)=M_{0}(0) e^{((k /(\nu+1))-1) t} .
\end{aligned}
$$

The long-time behavior of these moments is independent of the initial conditions and instead depends upon the exponent $\nu$ and the constant $k$. When $k<2(\nu+3), M_{2} \rightarrow \infty$ as 
$t \rightarrow \infty$, while, otherwise, $M_{2} \rightarrow 0$. The zeroth moment, however, depends upon $k$ and $\nu$ in a different way. When $k<\nu+1, M_{0} \rightarrow 0$ as $t \rightarrow \infty$ and $M_{0} \rightarrow \infty$ otherwise. Note that for $-1<\nu<0$ and $\nu+1<k<2(\nu+3)$, both $M_{0}$ and $M_{2} \rightarrow \infty$ as $t \rightarrow \infty$. This prediction is consistent with the following interpretation. If both $M_{0}$ and $M_{2} \rightarrow \infty$, the mass in the system partitions such that a bimodal particle size distribution evolves where some of the mass is dominated by fragmentation and breaks up into ever-smaller sizes (accounting for $M_{0} \rightarrow \infty$ ) while the rest of the mass in the system is dominated by coagulation (accounting for $M_{2} \rightarrow \infty$ ). For cases in which $M_{2} \rightarrow$ $\infty, M_{0} \rightarrow 0$ as $t \rightarrow \infty$ coagulation dominates the system, whereas if $M_{2} \rightarrow 0, M_{0} \rightarrow \infty$ fragmentation overpowers coagulation. The various regimes of the long-time behavior of $M_{0}$ and $M_{2}$ for these kernels is illustrated in Fig. 1.

(6) $\beta=0, K(x, y)=x y$. The equations for $M_{2}$ and $M_{0}$ for this case are

$M_{2}(t)$

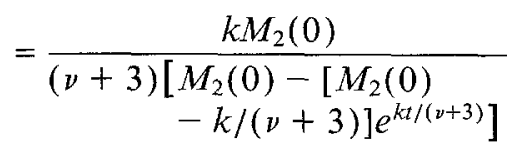

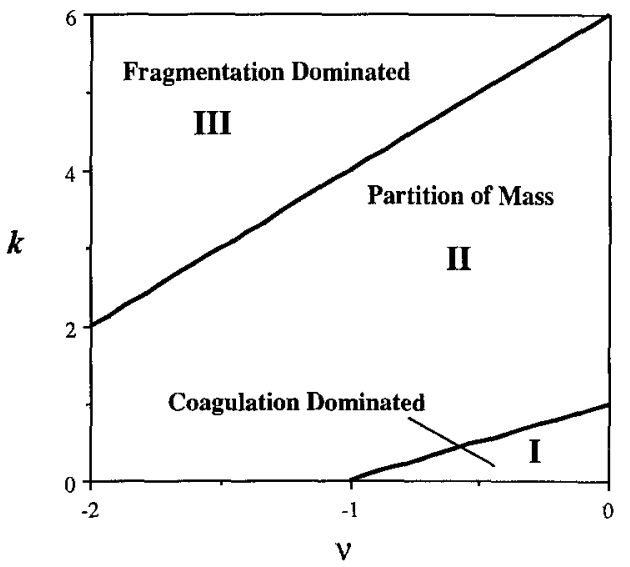

FIG. 1. Stability portrait for case 5 showing dependence on $k$ and $\nu$. In region I coagulation dominates at long times and $M_{2} \rightarrow \infty$ while $M_{0} \rightarrow 0$. In region II a bimodal distribution evolves and $M_{2}, M_{0} \rightarrow \infty$. Fragmentation dominates in region III and thus $M_{0} \rightarrow \infty$ while $M_{2} \rightarrow 0$.

Journal of Colloid and Interface Science, Vol. 133, No. 1, November 1989

$$
\begin{aligned}
M_{0}(t) & =\frac{(\nu+1)}{2 k} \\
& +\left[M_{0}(0)-\frac{(\nu+1)}{2 k}\right] e^{k t /(\nu+1)} \cdot[10 \mathrm{~b}]
\end{aligned}
$$

In this case, the long-time behavior of both $M_{2}$ and $M_{0}$ depends upon the initial conditions. $M_{2}$ diverges in a finite time, suggesting gelation, when $M_{2}(0)>k /(\nu+3)$, whereas $M_{2} \rightarrow 0$ as $t \rightarrow \infty$ if $M_{2}(0)<k /(\nu+3)$. When $M_{0}(0)>(\nu+1) / 2 k$ then $M_{0} \rightarrow \infty$ as $t \rightarrow \infty$. Otherwise $M_{0}$ becomes negative, which again is indicative of gelation. We note that, under certain initial conditions implied above, it is possible that $M_{0} \rightarrow \infty$ while $M_{2}$ diverges. As in case 5 above, we believe that this suggests that mass in the system partitions such that a bimodal size distribution evolves. For this set of kernels some of the mass is dominated by fragmentation while the rest of the mass undergoes a gelation transition. Figure 2 illustrates the various regimes for the long-time behavior of $M_{2}$ and $M_{0}$. Note that it appears that it is possible for $M_{0}$ to become negative while $M_{2} \rightarrow 0$. However, conservation of mass must be satisfied at the initial conditions, and thus this situation can never occur.

(7) $\beta=1, K(x, y)=1$. For cases in which $\beta=1$, Eq. [4] couples to higher order terms and prospects for obtaining explicit solutions become remote (except for $\nu=0$, as noted below). However, the zeroth moment couples to $M_{1}$, which is constant in the absence of phase transitions. Therefore, Eq. [4] yields

$$
M_{0}(t)=\alpha \frac{1+b e^{-(2 k /(\nu+1)) t}}{1-b e^{-(2 k /(\nu+1)) t}},
$$

where $\alpha \equiv \sqrt{2 k /(\nu+1)}$ and $b=\left(M_{2}(0)-\alpha\right) /$ $\left(M_{2}(0)+\alpha\right)$. The long-time behavior of [11] is independent of the initial conditions and always approaches the steady state, $M_{0} \rightarrow \alpha$ as $t \rightarrow \infty$. This result is consistent with the findings of Blatz and Tobolsky (23), who solved this problem explicitly for $c(x, t)$ for the case $\nu=0$ (binary breakup) and showed that a steady state is reached. 

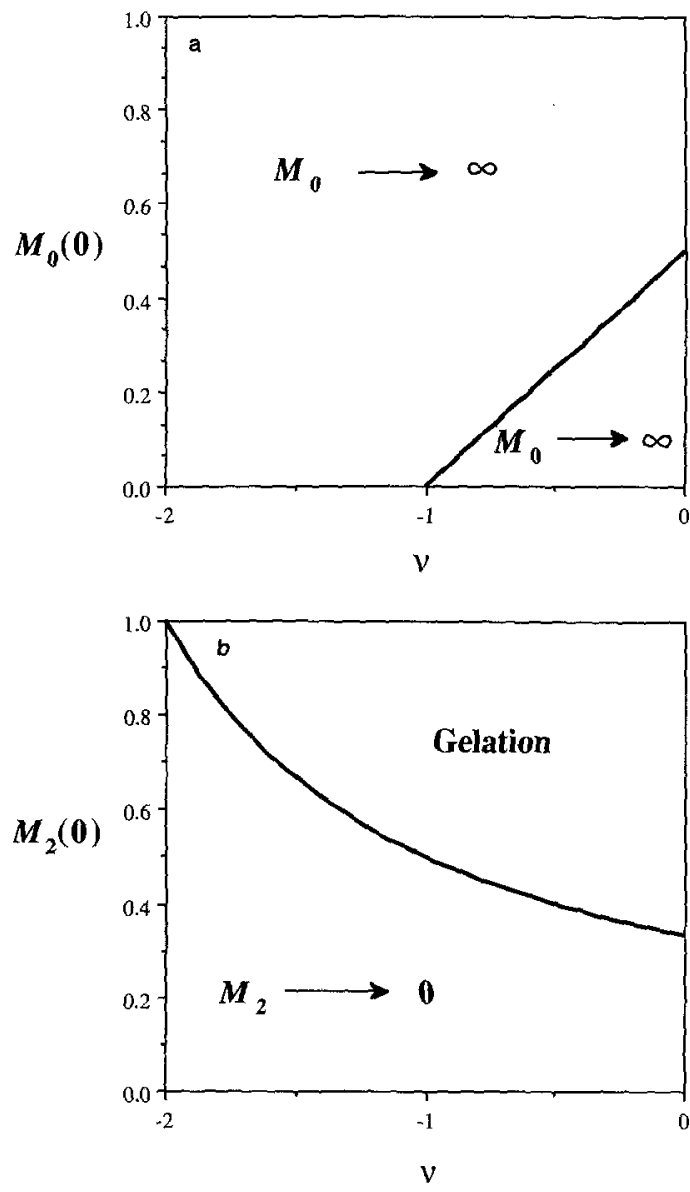

FIG. 2. Stability portraits for case $6(k=1)$. Since mass conservation must be satisfied for the initial conditions, these plots are not independent.

(8) $\beta=1, K(x, y)=x+y$. The zeroth moment is given by

$$
\begin{aligned}
M_{0}(t)= & \frac{k}{(\nu+1)} \\
& \quad+\left(M_{0}(0)-\frac{k}{(\nu+1)}\right) e^{-t},
\end{aligned}
$$

implying that $M_{0} \rightarrow k /(\nu+1)$ as $t \rightarrow \infty$. Therefore this combination of kernels appears to always lead to a steady state, at least for $\nu$ $>-1$.

(9) $\beta=1, K(x, y)=x y \cdot M_{0}$ is given by

$$
M_{0}(t)=M_{0}(0)+\left(\frac{k}{\nu+1}-\frac{1}{2}\right) t \text {. }
$$

When $k>(\nu+1) / 2, M_{0}$ grows linearly and it appears that at least some of the mass in the system must be dominated by breakup. It is not likely, however, that a bimodal size distribution occurs in this case, since the breakup rate becomes stronger as particles become larger. Rather, we believe that fragmentation simply overpowers coagulation in these cases and thus we conjecture that $M_{2} \rightarrow 0$. However, when $k<(\nu+1) / 2, M_{0}$ becomes negative in a finite time indicating that coagulation dominates the system and a gelation transition occurs.

\section{Discussion of Stability}

The significance of the preceding analyses is best shown in Table I, where we have summarized the behavior of Eq. [4] for each set of kernels and have arranged the nine cases by their homogeneity indices. A coagulation kernel is homogeneous of order $\lambda$ if $K(a x, a y)$ $=a^{\lambda} K(x, y)$. For the cases we consider, $\lambda=0$, 1,2 for the constant, sum, and product kernels, respectively. The homogeneity of the fragmentation rate $a(x)$ is simply $\beta$.

Note that the three sets of kernels in the upper right-hand corner of Table I always approach steady states, regardless of initial conditions or the value of the constant $k$ or exponent $\nu$, while no such steady states exist for the other six cases, which are therefore unstable. More specifically, it appears that only when $\lambda$ and $\beta$ obey

$$
\beta-\lambda+1>0
$$

do steady-state solutions occur. We have also performed a similar analysis of the moment equations for the parabolic breakup kernel in combination with each of the three basic coagulation kernels. Table II, which summarizes our findings for six combinations of kernels, is also consistent with Eq. [14]. Thus, although we have only verified [14] for fifteen specific cases, we expect that it is true in general for homogeneous rate kernels. Other evidence, based upon scaling assumptions, also supports 
TABLE I

Summary of Long-Time Behavior for Power-Law Breakup with Coagulation Breakup Rate Exponent, $\beta$

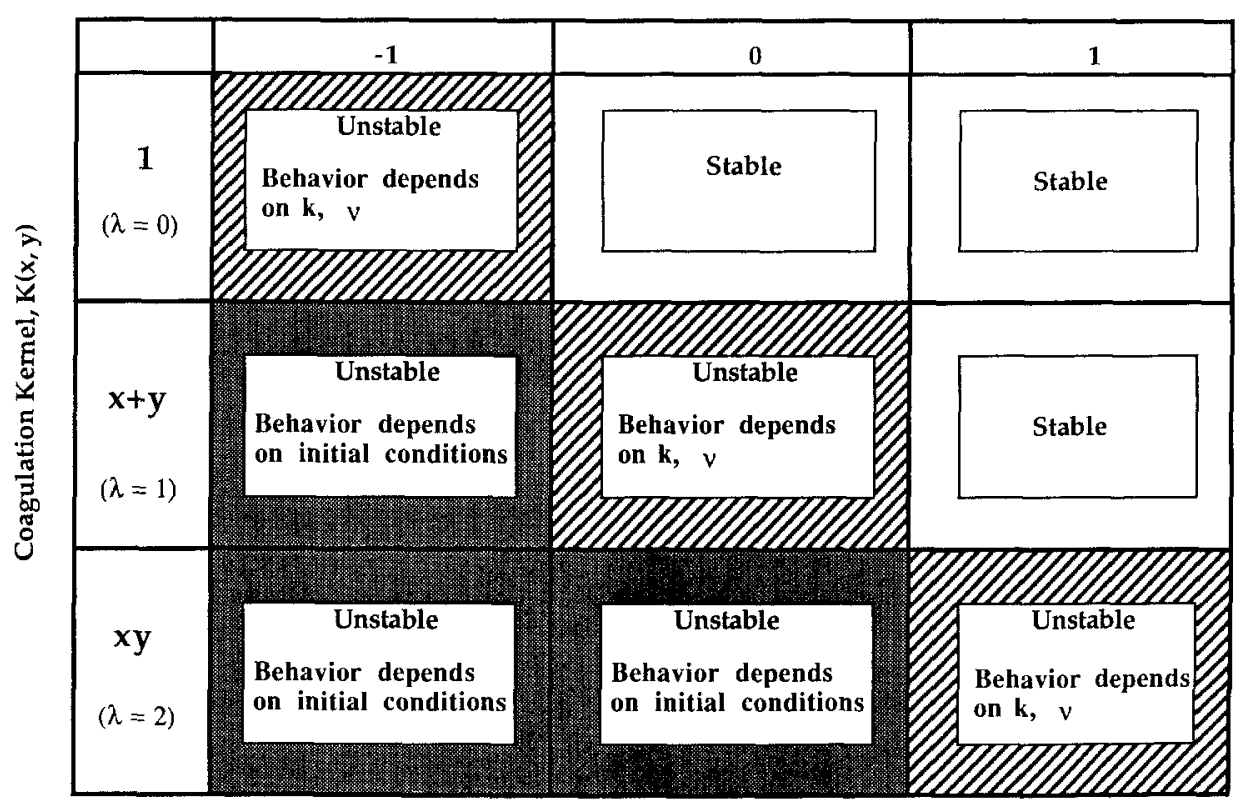

the validity of [14]. The homogeneity exponent $\beta$ is related to the binary fragmentation kernel, $F(x, y)$, by $a(x)=(1 / 2) \int_{0}^{x} F(y, x$ $-y) d y$. Thus, it may be shown that the scaling relation given by Family et al. (18), which applies to coagulation with binary fragmentation at steady state, also requires that [14] be satisfied. Furthermore, Eq. [14] is equivalent to the stability criterion given by Sorensen et al. (19) for coagulation-binary fragmentation. However, as noted previously, their findings were based upon a faulty assumption $(21,22)$.

Lastly, we find that Tables I and II show that when $\beta-\lambda+1=0$ (unstable cases bordering stable cases), the long-time behavior of the system (whether the size distribution evolves toward ever-increasing sizes, ever-decreasing sizes, or a bimodal distribution ) does not depend upon the initial conditions, but instead depends only upon the constant $k$ and, for the power-law breakup kernel, also upon the exponent $\nu$.

Joumal of Colloid and Interface Science, Vol, 133, No. 1, November 1989

\section{SCALING SOLUTIONS}

Meakin and Ernst (22) give the two-parameter scaling law

$$
\begin{aligned}
c(x, t, k)=x^{-2} f(u, T) & \\
x, t & \rightarrow \infty, k \rightarrow 0,
\end{aligned}
$$

where again $k$ is a constant representing the relative strength of fragmentation to coagulation, $u=x / S(t, k), T=t k^{X}$, and $S(t, k)$ is the characteristic particle size, such that $T$ remains constant in the scaling limit and $S(\infty$, $k$ ) $\sim k^{-Y}$ (note that we use $X$ and $Y$ as exponents while $x$ and $y$ refer to size variables). By requiring that $f(u, 0)$ reduces to the irreversible coagulation scaling form $(20)$ and that $f(u, \infty)$ gives the scaling law for coagulationfragmentation at steady state (18), a generalized scaling description is obtained. However, the scaling limit $x, t \rightarrow \infty, k \rightarrow 0$ implies that [15] only applies to coagulation-fragmentation systems that reach steady states, such that the steady state is approached from 
TABLE II

Summary of Long-Time Behavior for Parabolic Breakup with Coagulation

Breakup Rate Exponent, $\beta$

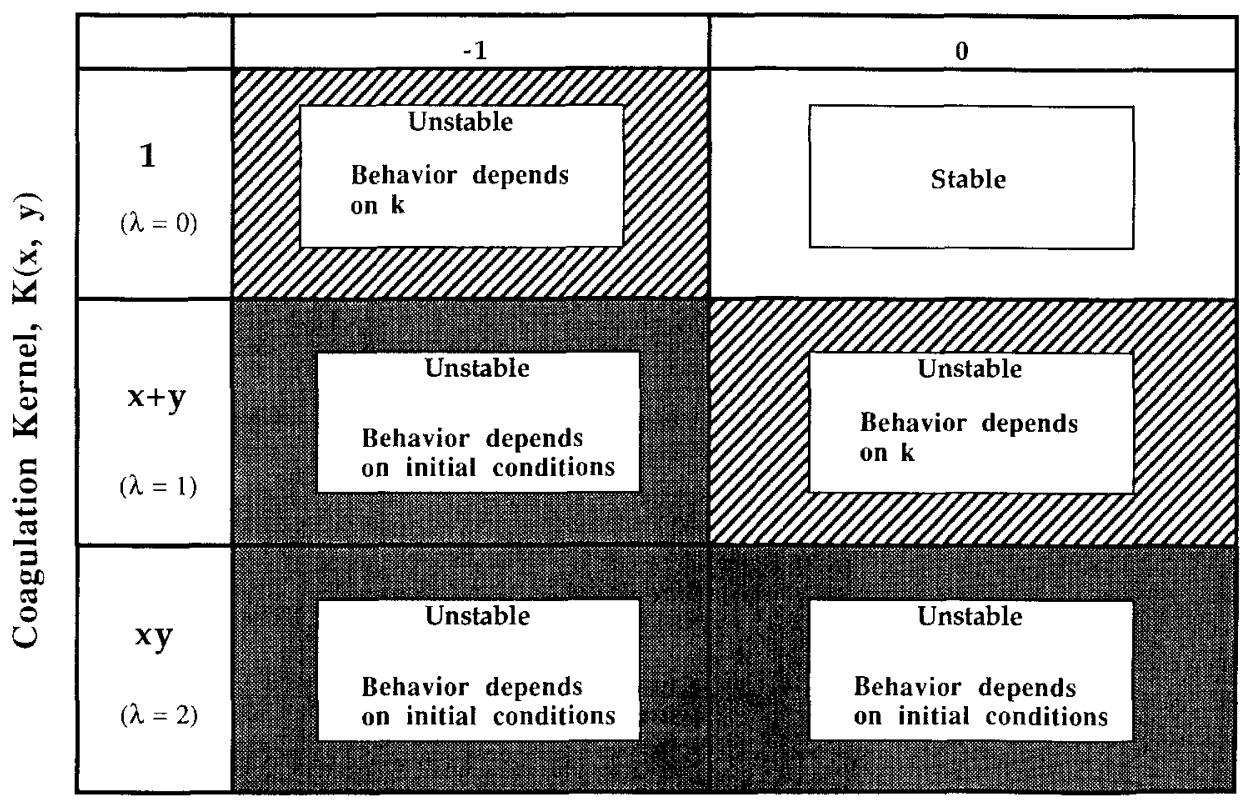

below (coagulation dominates the short-time behavior of the system ).

It may be shown that this two-parameter scaling law also applies to the generalized Eq. [3]. Equation [15] implies the similarity solution

$$
c(x, t, k)=k^{2 Y} c\left(x k^{Y}, t k^{X}, 1\right) .
$$

The breakup distribution function, $b(x \mid y)$, may be written as

$$
b(x \mid y)=y^{-1} b^{\prime}\left(\frac{x}{y}\right)
$$

for both the power-law and parabolic breakup kernels, as well as for most other breakup kernels of interest. Substitution of [16], [17], and the homogeneity relation $K(x / s, y / s)$ $=s^{-\lambda} K(x, y)$ into [3] leads to

$k^{2 Y+X} \frac{\partial c(u, T)}{\partial T}$

$$
\begin{gathered}
=k^{(3-\lambda) Y}\left[\frac{1}{2} \int_{0}^{u} K(v, u-v) c(v, T)\right. \\
\times c(u-v, T)-c(u, T) \int_{0}^{\infty} \\
\times K(u, v) c(v, t)] d v-k^{1+Y(2-\beta)} \\
\times\left[a(u) c(u, T)-\int_{u}^{\infty} a(v)\right. \\
\left.\times b^{\prime}\left(\frac{u}{v}\right) c(v, T) d v\right],
\end{gathered}
$$

where $u=x k^{Y}$ and $v=y k^{Y}$. Therefore, [16] is a similarity solution to [3] if $Y=(\beta-\lambda$ $+1)^{-1}$ and $X=Y(1-\lambda)$. This result is a generalization of the findings of Meakin and Ernst (22), who considered binary fragmentation with $F(x, y)=(x+y)^{\alpha}$, where the relation $a(x)=(1 / 2) \int_{0}^{x} F(y, x-y) d y$ implies $\beta=\alpha+1$.

Journal of Colloid and Interface Science, Vol. 133, No. I, November 1989 
When [16] is a solution to [3], the moments of the size distribution should scale as

$$
M_{n}(t, k)=k^{Y(1-n)} M_{n}(T, 1) .
$$

For example, consider the power-law breakup kernel with $\beta=0$ in combination with the constant coagulation kernel, $K(x, y)=1(\lambda$ $=0$ ), where the explicit solutions for $M_{2}$ and $M_{0}$ are given by [8] and approach a steady state as $t \rightarrow \infty$. Thus, $Y=1, X=1$, and [19] gives

$$
\begin{aligned}
& M_{2}(t, k)=k^{-1} M_{2}(t k, 1) \\
& M_{0}(t, k)=k M_{0}(t k, 1) .
\end{aligned}
$$

Comparison of [20] with the explicit solution [8] shows that the scaling law [19] is indeed satisfied for these kernels, where

$$
\begin{aligned}
& M_{2}(T, 1)=(\nu+3)\left(1-e^{-T /(\nu+3)}\right) \quad[21 \mathrm{a}] \\
& M_{0}(T, 1)=\frac{2 k}{(\nu+1)\left(1-e^{-T /(\nu+1)}\right)} \cdot[21 \mathrm{~b}]
\end{aligned}
$$

A similar exercise may be performed for the other two combinations of kernels which have steady-state solutions (cases 7 and 8 considered in section II).

\section{CONCLUSION}

We have presented specific moment solutions to a generalized coagulation-fragmentation equation and have examined the implications that these solutions have for the stability of these systems. Our results are consistent with the criterion $\beta-\lambda+1>0$ for stable steady-state size distributions to exist. For homogeneous rate kernels we find that the existence of steady states does not depend upon the initial conditions or upon the value of a constant multiplying the coagulation or fragmentation kernel (such as $k$ ), but rather it depends only upon the homogeneity of the kernel. We have verified this stability criterion for 15 specific cases and have shown that it is consistent with the findings of other investigators who considered coagulation with binary fragmentation. Thus, we believe that it may be used to predict the stability behavior for other homogeneous rate kernels or for rate kernels that become homogeneous asymptotically at large sizes. Finally, we have shown that the scaling equation [15], which is valid for coagulation with binary fragmentation, also applies to the more general PBE [3].

\section{ACKNOWLEDGMENT}

We acknowledge support from National Science Foundation Grant DMR-8619731 for this research.

\section{REFERENCES}

1. von Smoluchowski, M., Physik Z. 17, 557, 568, 585 (1916).

2. Golovin, A. M., Izv. Geophys. Ser. 5, 783 (1963).

3. Saffman, P. G., and Turner, J. S., J. Fluid. Mech. 1, 16 (1956).

4. McLeod, J. B., Proc. London Math. Soc. 3rd Ser. 14, 445 (1964).

5. Ziff, R. M., J. Stat. Phys. 23, 241 (1980).

6. van Dongen, P. G. J., and Ernst, M. H., J. Stat. Phys. 44, 785 (1986).

7. Montroll, E. W., and Simha, R., J. Chem. Phys. 8, 721 (1940).

8. Ballauff, M., and Wolf, B. A., Macromolecules 14, 654 (1981).

9. Basedow, A. M., Ebert, K. H., and Ederer, H. J., Macromolecules 11, 774 (1978).

10. Charlesby, A., Proc. R. Soc. (London) A 224, 120 (1954).

11. Austin, L., Powder Technol. 5, 1 (1971).

12. Peterson, T. W., Aerosol. Sci. Technol. 5, 93 (1986).

13. Fillipov, A. F., "Theory Probability: Its Application" (English transl.), Vol. 4, p. 275. 1961.

14. Kerstein, A. R., and Edwards, B. F., Chem. Eng. Sci. 42, 1629 (1987).

15. Dunn-Rankin, D., and Kerstein, A. R., Combust. Flame 69, 193 (1987).

16. McGrady, E. D., Ph.D. thesis, The University of Michigan, 1988.

17. McGrady, E. D., and Ziff, R. M., Phys. Rev. Lett. 58, 892 (1987).

18. Family, F., Meakin, P., and Deutch, J. M., Phys. Rev. Lett. 57, 727 (1986).

19. Sorensen, C. M., Zhang, H. X., and Taylor, T. W., Phys. Rev. Lett. 59, 363 (1987).

20. Viscek, T., and Family, F., Phys. Rev. Lett. 52, 1669 (1984).

21. Vigil, R. D., and Ziff, R. M., Phys. Rev. Lett. 61, 1431 (1988).

22. Meakin, P., and Ernst, M. H., Phys. Rev. Lett. 60, 2503 (1988).

23. Blatz, P. J., and Tobolsky, A. V., J. Phys. Chem. 49, 77 (1945).

24. van Dongen, P. G. J., and Ernst, M. H., J. Stat. Phys. 37, 301 (1985). 